The Astrophysical Journal, 210:L39-L43, 1976 November 15

(c) 1976. The American Astronomical Society. All rights reserved. Printed in U.S.A.

\title{
THE NATURE OF THE BROAD MOLECULAR LINE EMISSION AT THE KLEINMANN-LOW NEBULA
}

\author{
JoHN KWAN \\ State University of New York, Stony Brook \\ AND \\ Nick Scoville \\ University of Massachusetts, Amherst \\ Received 1976 July 8
}

\begin{abstract}
We have mapped the high-velocity emission in the wings of the $\mathrm{CO}$ line at the Kleinmann-Low infrared nebula. The half-power diameter is found to be $80^{\prime \prime}$. Upon analysis of the observed intensity profiles and the variations in angular size at different velocities, we conclude that a differentially expanding envelope, possibly the result of an explosive event, is the most likely explanation. The kinetic energy in the flow is $4 \times 10^{47} \mathrm{ergs}$, based on a numerical model of the $\mathrm{CO}$ emission. The recently observed $v=1 \rightarrow 0$ radiation of $\mathrm{H}_{2}$ would arise from the shock front preceding the expanding gas.

Subject headings: interstellar: molecules - nebulae: individual
\end{abstract}

\section{INTRODUCTION}

An extraordinary feature of the millimeter molecular lines observed at the Kleinmann-Low nebula is the very large velocity extent of the emission. At this position, several molecules radiate over a range of at least $40 \mathrm{~km} \mathrm{~s}^{-1}$; and in the $\mathrm{CO}$ observations presented below, gas over a full range of $120 \mathrm{~km} \mathrm{~s}^{-1}$ is clearly present (see also Kuiper, Zuckerman, and Kuiper 1975). Though this high-velocity emission of $\mathrm{CO}$ constitutes approximately $2 / 5$ of the total integrated line intensity at KL, its presence and analysis were largely overlooked because of the much stronger and narrower central peak at $v_{\mathrm{LSR}}=9 \mathrm{~km} \mathrm{~s}^{-1}$. This broad molecular emission was first apparent in an $\mathrm{H}_{2} \mathrm{~S}$ line (Thaddeus et al. 1972), and became especially prominent in subsequent observations of $\mathrm{SO}$ and $\mathrm{SO}_{2}$ (Gottlieb and Ball 1973; Snyder et al. 1975). Further evidence for this emission is present in the HCN and SiO data (Wannier et al. 1974; Dickinson et al. 1976).

A most important observational constraint on understanding the nature of this high-velocity gas is the size of the region in which it is present, as it greatly affects the deduced energetics of the phenomenon and the physical conditions of the region. Here we report observations made on the National Radio Astronomy Observatory $^{1}$ (NRAO) 36 foot $(11 \mathrm{~m})$ antenna in 1976 May, which aim to determine this size and to obtain good profiles of the $\mathrm{CO}$ and ${ }^{13} \mathrm{CO} J=1 \rightarrow 0$ lines. These observational results are presented in the next section. In \& III theoretical considerations based on these results are discussed; and a model in which gas is expanding radially with $v \propto r$, possibly the result of an explosion, is put forward. Implications of this model are discussed in $\$ I V$.

${ }^{1}$ Operated by Associated Universities, Inc., under contract with the National Science Foundation.

\section{OBSERVATIONS}

Carbon monoxide spectra were obtained at positions spaced $30^{\prime \prime}$ apart on a $90^{\prime \prime} \times 90^{\prime \prime}$ grid centered on the KL position $\left(\alpha_{1950}=5^{\mathrm{h}} 32^{\mathrm{m}} 47^{\mathrm{s}}, \delta_{1950}=-5^{\circ} 24^{\prime} 21^{\prime \prime}\right)$. The two filter banks chosen provided simultaneous coverage of 166 and $333 \mathrm{~km} \mathrm{~s}^{-1}$ at resolutions of 0.6 and 1.2 $\mathrm{km} \mathrm{s}^{-1}$, respectively. Spectra of the $\mathrm{CO}$ and ${ }^{13} \mathrm{CO}$ emission at $\mathrm{KL}$ are presented in Figure 1a. The CO spectrum is replotted in Figure $1 b$ on a finer intensity scale, and a spectrum at $60^{\prime \prime} \mathrm{S}$ is also shown. The emission is centered at an LSR velocity of $\sim 9 \mathrm{~km} \mathrm{~s}^{-1}$ and extends over a range of at least $100 \mathrm{~km} \mathrm{~s}^{-1}$ (henceforth all velocities $v$ will be referred relative to the central velocity). The intensity in the line wings of the emission at $\mathrm{KL}$ falls off on both sides approximately as $v^{-1}$ between 10 and $20 \mathrm{~km} \mathrm{~s}^{-1}$ and as $v^{-2}$ between 20 and $50 \mathrm{~km} \mathrm{~s}^{-1}$ (Fig. 1a). At all observed positions the emission is quite symmetric about the central velocity. When the mean velocity, defined as

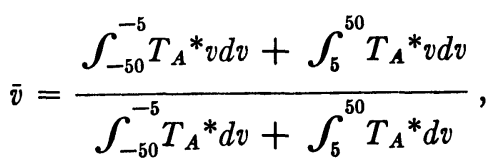

is averaged over the central 10 observed positions, it is about $-2 \mathrm{~km} \mathrm{~s}^{-1}$.

The spatial dependence of the high velocity emission is indicated in Figure 2a. Contours of the mean line intensity, $\left\langle T_{A}^{*}\right\rangle\left(=\int T_{A}^{*} d v / \mathcal{S} d v\right)$, were calculated from the spectra with the limits of integration, $v=$ -40 to $-10 \mathrm{~km} \mathrm{~s}^{-1}$ and $v=10$ to $40 \mathrm{~km} \mathrm{~s}^{-1}$, chosen to exclude the central peak. The diameter of the region measured at $\frac{1}{2}$ peak $\left\langle T_{A}{ }^{*}\right\rangle$ is about $80^{\prime \prime}$. The decrease in intensity with distance is very abrupt beyond this half-power contour. It is clear from the half-power size 

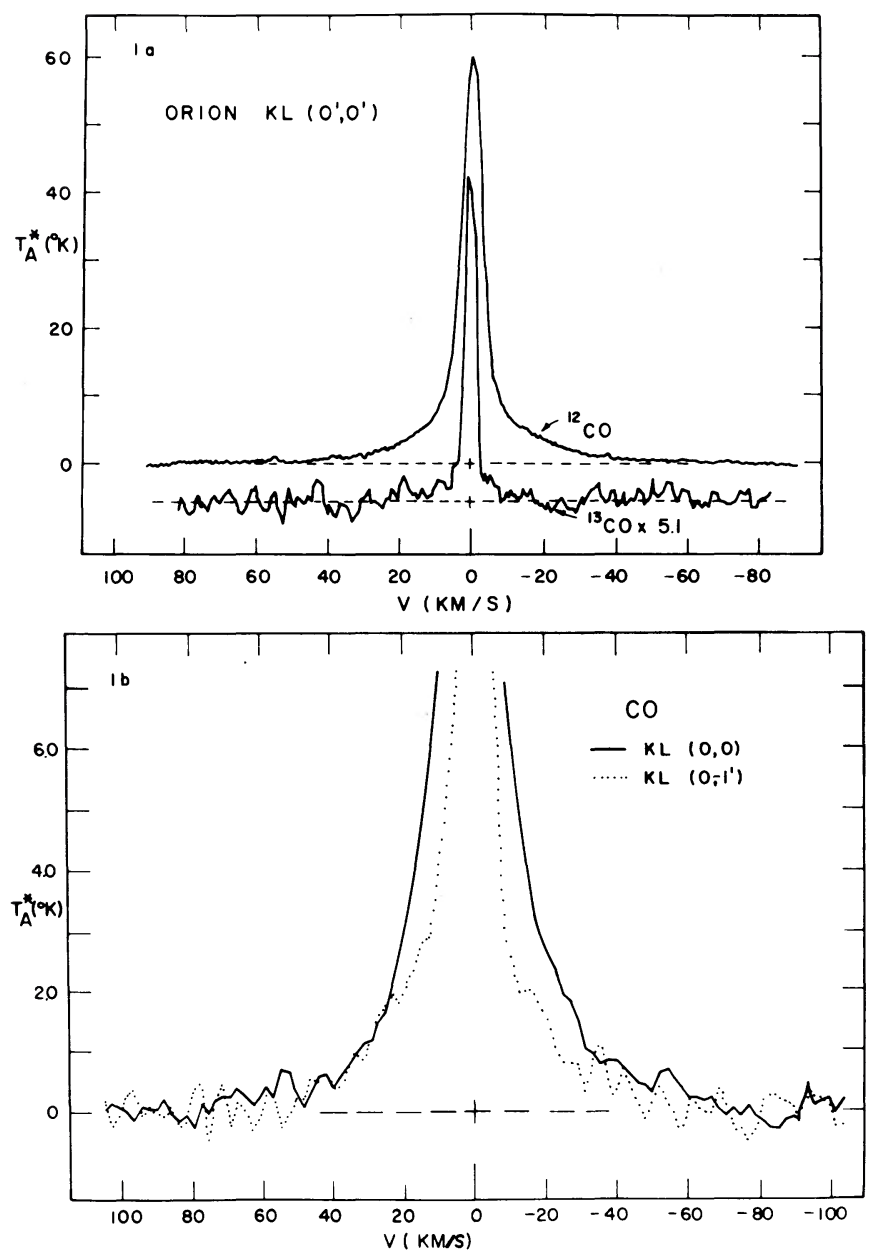

Fig. 1a.-Spectra of the $\mathrm{CO}$ and ${ }^{13} \mathrm{CO} J=1 \rightarrow 0$ emission at the Kleinmann-Low nebula $\left(\alpha_{1950}=5^{\mathrm{b}} 32^{\mathrm{m}} 47^{\mathrm{s}}, \delta_{1950}=-5^{\circ} 24^{\prime} 21^{\prime \prime}\right)$. The ordinate indicates antenna temperature after correction for antenna and atmospheric losses; the abscissa is radial velocity relative to $v_{\mathrm{LSR}}=$ $9 \mathrm{~km} \mathrm{~s}^{-1}$.

Fig. 1b. - Spectra of the $\mathrm{CO}$ emission in the line wings at $\mathrm{KL}$ and at a position $60^{\prime \prime} \mathrm{S}$. Note the relatively stronger emission at progressively larger $|v|$ at the $60^{\prime \prime} \mathrm{S}$ position.

that the high-velocity emission cannot arise from a region small compared with the antenna beam $(\mathrm{HPBW} \approx$ $\left.66^{\prime \prime}\right)$, since the response of the antenna to a point source displaced $45^{\prime \prime}$ away would be $\sim \frac{1}{4}$ the peak response (Ulich and Haas 1976), and yet we measure close to $\frac{1}{2}$ peak intensity.

Comparison of the spectrum at $\mathrm{KL}$ with that at $60^{\prime \prime} \mathrm{S}$ indicates that, as one moves off $\mathrm{KL}$, the emission in the line wings decreases more slowly at progressively larger $|v|$. This is a characteristic of most off-center spectra, and is illustrated in Figure $2 b$ which shows the ratio of the emission between $|v|=20$ and $40 \mathrm{~km} \mathrm{~s}^{-1}$ to that between $|v|=10$ and $20 \mathrm{~km} \mathrm{~s}^{-1}$. While it is difficult to measure the size of the emission at the highest velocities, we can conclude that the extent of the emission at low velocities is not larger than that at high velocities.

An indication of the relatively low opacity of the $\mathrm{CO}$ emission in the line wings may be obtained from comparison of the $\mathrm{CO}$ and ${ }^{13} \mathrm{CO}$ profiles at $\mathrm{KL}$ (Fig. 1a). At $|v|=10 \mathrm{~km} \mathrm{~s}^{-1}$ the $\mathrm{CO}:{ }^{13} \mathrm{CO}$ intensity is at least $20: 1$, in contrast to the corresponding ratio of $6: 1$ at the central peak.

\section{THEORETICAL ANALYSIS}

We do not believe the broad-band emission is a superposition of many narrow lines from sources at widely different Doppler velocities. Though the $\mathrm{H}_{2} \mathrm{O}$ maser lines observed at $\mathrm{KL}$ span a velocity range of $\sim 30 \mathrm{~km} \mathrm{~s}^{-1}$ (Sullivan 1971), the width, smoothness, shape, and high degree of constancy of the CO profile over a $2^{\prime}$ region all argue against a fine structure model for the millimeter line emission. We believe systematic, large-scale motion must be responsible for the large line width. There are three kinds of such motion: rotation, collapse, and expansion. We shall argue, based solely on the observed properties of the emission outlined in $\S \mathrm{II}$, that a differentially expanding envelope is the most likely explanation of the observations.

The lack of obvious asymmetry in any of the observed profiles argues strongly against rotation as being 


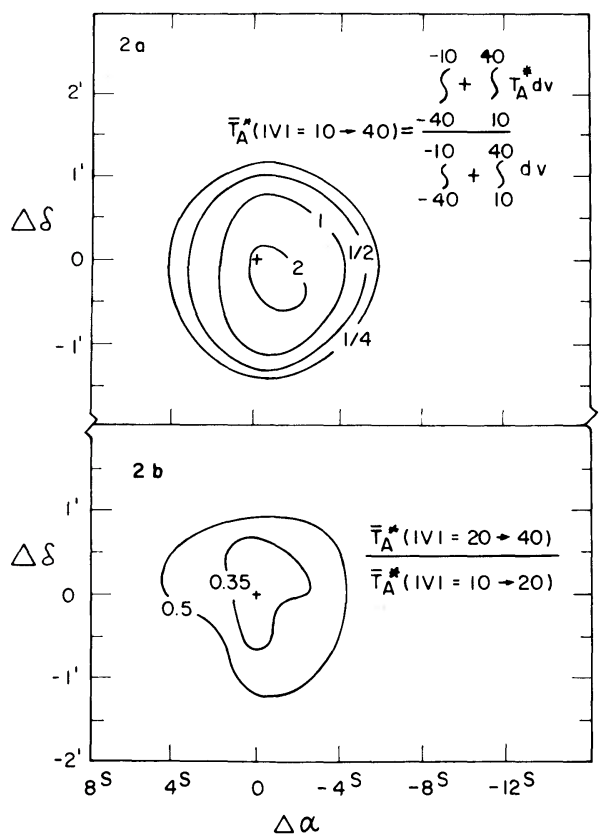

Fig. 2a.-Contours of the mean antenna temperature $(\mathbf{K})$ in the line wings as a function of right ascension and declination displacements from KL. The limits of integration were chosen to exclude emission from the central narrow peak at $v_{\mathrm{LSR}}=9 \mathrm{~km} \mathrm{~s}^{-1}$.

FIG. 2b.-The ratio of $\left\langle T_{A}{ }^{*}\right\rangle$ at high $|v|$ in the line wings to $\left\langle T_{A}{ }^{*}\right\rangle$ at low $|v|$ exhibits a systematic increase away from the KL position.

responsible for the large line width. Though the contour maps indicate that the region is larger than the beam size, profiles taken at diametrically opposite points are very similar and show no evidence of a radial velocity gradient.

The profile shapes may be used to rule out both collapse and expansion models in which the motion is at a constant velocity. For such a motion of magnitude $v_{r}$ in an unresolved source, it can be shown that the intensity profile is flat-topped from $v=-v_{r}$ to $+v_{r}$ if the emission is optically thin, and is a centrally peaked conic $\left\{T_{A}(v) \propto\left(1-v^{2} / v_{r}^{2}\right)\right\}$, if the emission is optically thick. In the more relevant case of a resolved envelope, the intensities near the edges $v= \pm v_{r}$ are enhanced over those near $v=0$ (e.g., Kuiper et al. 1976). All of these profile shapes are very different from the observed ones.

For a differentially collapsing envelope the velocity can be either a decreasing or an increasing function of radius. The argument against the first case is provided by the observational result that the size of the emission at a larger velocity is greater than that at a lower velocity. To illustrate, in the case of $v_{r} \propto r^{-1 / 2}$, the region of emission at a given line-of-sight velocity $v$ projects onto the plane of the sky a diameter proportional to $v^{-2}$. The observations show that at $|v|=30$ $\mathrm{km} \mathrm{s}^{-1}$ the emission region has a diameter of $\sim 80^{\prime \prime}$. With the above velocity distribution, the linear dimension of the emission at $|v|=10 \mathrm{~km} \mathrm{~s}^{-1}$ should then be 9 times greater. Yet it is observed to be actually slightly smaller.
If, on the other hand, the collapse velocity is an increasing function of radius, and is $30 \mathrm{~km} \mathrm{~s}^{-1}$ at $3 \times 10^{17} \mathrm{~cm}$ (corresponding to $45^{\prime \prime}$ at a distance of $500 \mathrm{pc}$ ), then it is difficult to understand the observed abrupt falloff in the intensity of the high-velocity emission beyond $60^{\prime \prime}$ from the $\mathrm{KL}$ position.

If the large velocity range in the $\mathrm{CO}$ emission is due to an expansion, the motion must be differential, and the most logical velocity structure is $v_{r}=v_{0} r / r_{0}$, since the gas, except at the front, must be approximately in free expansion. Such a flow can occur as a result of an explosive event. With this velocity distribution, the contour at an observed velocity $v$ is described by

$$
r^{2}=p^{2}+r_{0}^{2}\left(\frac{v}{v_{0}}\right)^{2}
$$

where $p$, the impact parameter, is the perpendicular distance between the line of sight and the center of expansion. From equation (1) it is clear that the differences among the emission at various velocities decrease with increasing $p$, for the assumed velocity law in a spherically symmetric model. This model will therefore account for the larger half-power size at $v=$ $30 \mathrm{~km} \mathrm{~s}^{-1}$ than at $v=15 \mathrm{~km} \mathrm{~s}^{-1}$ (Fig. $2 b$ ).

A complete model must calculate the density distribution after the propagation of a strong shock through the medium, and the temperature distribution from consideration of the heating and cooling processes as the gas expands. In this Letter, however, we shall adopt reasonable power-law forms for both the temperature and density distributions, as the most important parametrization is the velocity structure. In Figure 3 we present a numerical model in which

$$
\begin{aligned}
v_{r} & =10 \mathrm{~km} \mathrm{~s}^{-1}\left(\frac{r}{r_{0}}\right), \\
n_{\mathrm{H}_{2}}(r) & =10^{6} \mathrm{~cm}^{-3}\left(\frac{r_{0}}{r}\right)^{3}, \\
T_{K}(r) & =50 \mathrm{~K}\left(\frac{r_{0}}{r}\right)^{1 / 2}, \quad r \leq 6 r_{0},
\end{aligned}
$$

with $r_{0}=10^{17} \mathrm{~cm}$. A $[\mathrm{CO}] /\left[\mathrm{H}_{2}\right]$ ratio of $2.5 \times 10^{-5}$ was assumed; and, in view of the high gas density, the excitation temperature was set equal to the kinetic temperature $T_{K}$ at all radii. The source brightness distribution was convolved with the antenna beam pattern of Ulich and Haas (1976), namely, a HPBW of the main beam of $66^{\prime \prime}$ at $115 \mathrm{GHz}$ and an error beam of $25^{\prime}$. The convolved intensity profiles at beam displacements of $0^{\prime \prime}, 45^{\prime \prime}$, and $90^{\prime \prime}$ from the center are plotted. The observed profiles at $\mathrm{KL}$ and at $60^{\prime \prime} \mathrm{S}$ are also shown.

In this differential expansion model the parameters $v_{r}, n_{\mathrm{H}_{2}}$, and $T_{K}$ at $r_{0}$ are remarkably well constrained by the observations. The observed velocity extent and abrupt falloff in intensity beyond $60^{\prime \prime}$ from the center determine the choice of $v_{0}$ and $r_{0}$. The weakness of the ${ }^{13} \mathrm{CO}$ emission (Fig. 1a) indicates that the $\mathrm{CO}$ emission 


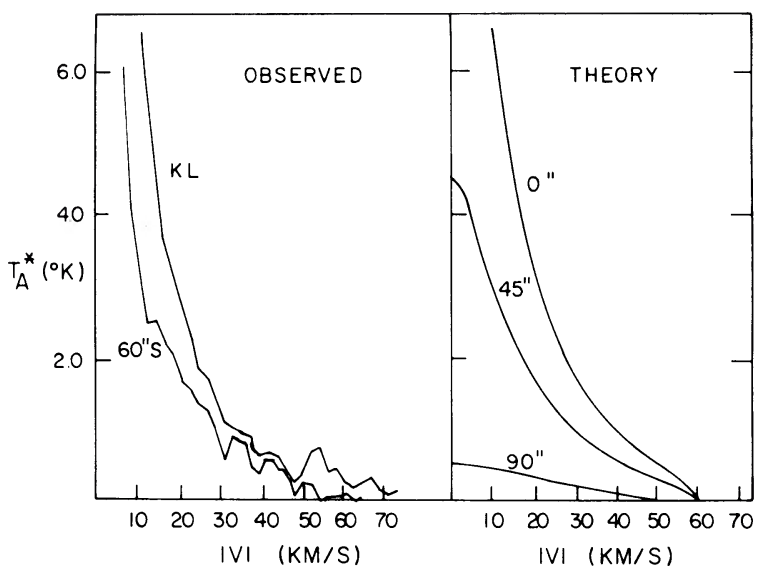

Fig. 3.-The observed $\mathrm{CO}$ line profiles at $\mathrm{KL}$ and at $60^{\prime \prime} \mathrm{S}$ are compared with theoretical line profiles determined at displacements of $0^{\prime \prime}, 45^{\prime \prime}$, and $90^{\prime \prime}$ from the center of expansion. The observed profiles shown are averages of the negative and positive velocity emission in Fig. $1 b$ (at $|v|<10 \mathrm{~km} \mathrm{~s}^{-1}$ they contain large contributions from the central narrow component at $v_{\mathrm{LSR}}=9 \mathrm{~km}$ $\left.\mathrm{s}^{-1}\right)$.

has optical depths of less than a few. With this information, plus an assumed $[\mathrm{CO}] /\left[\mathrm{H}_{2}\right]$ abundance ratio, the choice of $n_{\mathrm{H}_{2}}$ and $T_{K}$ at $r_{0}$ are constrained by the observed intensity. One might question the value of $T_{K}=50 \mathrm{~K}$ at $10^{17} \mathrm{~cm}$ as being too low, since the narrow central peak of the CO line, presumably arising from a larger region, has a brightness temperature of $\sim 90 \mathrm{~K}$ (Ulich and Haas 1976). It should be clear, however, that the relevant heating and cooling processes are entirely different in the two regions. Indeed, the temperature of the gas in the flow evolves with time while in the larger cloud there is probably a quasi-equilibrium between the rates of heating and cooling. In the expanding envelope the rate of cooling by $\mathrm{CO}$ emission alone $\left(5 \times 10^{-20}\right.$ ergs s$~^{-1} \mathrm{~cm}^{-3}$ at $r_{0}$ ) leads to a cooling time about equal to the expansion time.

The ${ }^{13} \mathrm{CO} / \mathrm{CO}$ intensity ratio, $\leq 1 / 20$, measured in the line wings at $v=10 \mathrm{~km} \mathrm{~s}^{-1}$ is probably the lowest such ratio observed at any source. A higher-quality ${ }^{13} \mathrm{CO}$ spectrum than we have obtained will place a strong limit on the $\mathrm{CO} /{ }^{13} \mathrm{CO}$ isotope ratio. In this regime of low opacity, it is expected that the $J=2 \rightarrow 1$ line will be even stronger in the line wings. With the above numerical model we calculate the $J=2 \rightarrow 1$ to $J=$ $1 \rightarrow 0$ intensity ratio to be 3.2 at $10 \mathrm{~km} \mathrm{~s}^{-1}$ (we have assumed that the telescope parameters at $230 \mathrm{GHz}$ are the same as those at $115 \mathrm{GHz}$, except that the HPBW of the diffraction beam is $33^{\prime \prime}$ instead of $66^{\prime \prime}$ ). This stronger emission in $J=2 \rightarrow 1$ was observed by Wannier and Phillips (1976).

\section{DISCUSSION}

We have shown that solely on the basis of the observational data a differential expansion model is warranted for the high-velocity emission. Here we consider several theoretical arguments that also pertain to this question.

Models invoking collapse, rotation, or radiation pressure to account for a velocity of $30 \mathrm{~km} \mathrm{~s}^{-1}$ at a radius of $3 \times 10^{17} \mathrm{~cm}$ require either a very large central mass or a luminosity far exceeding that presently observed. If the velocities are produced gravitationally (collapse or rotation), the mass within $3 \times 10^{17} \mathrm{~cm}$ must be $10^{4} M_{\odot}$. To evaluate the viability of radiation pressure to produce the observed motion, we first estimate the momentum of the flow. A lower limit to the mass giving rise to the broad-band emission can be obtained by assuming that the $\mathrm{CO}$ emission is optically thin. Using an averaged excitation temperature of $30 \mathrm{~K}$, and a $[\mathrm{CO}] /\left[\mathrm{H}_{2}\right]$ abundance ratio of $3 \times 10^{-5}$, the lower limit is $\sim 10 M_{\odot}$ (the actual mass is probably not far above this limit). In order to provide the total momentum observed $\left(10 M_{\odot} \times 50 \mathrm{~km} \mathrm{~s}^{-1}\right)$ in the expansion time of $\sim 3 \times 10^{3} \mathrm{yr}$, the rate of momentum transfer to the gas must be $8 \times 10^{6} L_{\odot} / c$. The required luminosity is about 80 times greater than what is observed at the Kleinmann-Low nebula (Werner et al. 1976).

A common difficulty encountered in any expansion model is the associated short time scale, $\sim 3 \times 10^{3} \mathrm{yr}$, of the phenomenon. This is of course short compared with the age of the molecular cloud, and the probability of seeing such an event in a given cloud must therefore be small.

Recently the $v=1 \rightarrow 0$ vibration rotational transitions of $\mathrm{H}_{2}$ at $\sim 2 \mu$ have been detected at the KL region (Gautier et al. 1976). From the mapping of this emission out to $\frac{1}{4}$ the peak intensity, the rate of energy loss due to the $S(1)$ line alone is calculated to be $\sim 1 L_{\odot}$ (Joyce and Grasdalen 1976). The total energy loss rate due to all presently observed lines must be $\sim 10 L_{\odot}$, an estimate which has not yet allowed for possible dust extinction. This strong $\mathrm{H}_{2}$ emission is likely to be excited by collisions at a high temperature, based on consideration of the relative intensities of the observed lines (Gautier et al. 1976). Such a strong $v=1 \rightarrow 0 \mathrm{H}_{2}$ emission would be produced, within the model we propose for the broad-band CO emission, at the shock front preceding the expanding gas where strong heating would occur. The $\mathrm{H}_{2}$ emission will come from a thin shell at a radius of $6 \times 10^{17} \mathrm{~cm}$. If the ambient gas density is $\sim 5 \times 10^{3} \mathrm{~cm}^{-3}$, the rate of heat input at the shock is $\sim 500 L_{\odot}$, which is more than enough to account for the rate of energy loss in the $v=1 \rightarrow 0$ lines and in the $v=0$ rotational lines at $T_{K}=2000 \mathrm{~K}$, even allowing for moderate extinction by dust at $2 \mu$. The contribution from the hot gas in the thin shell to the $\mathrm{CO}$ emission is insignificant, both because of the small amount of hot gas, and because of the narrow thickness of the shell.

A test of the model could be provided by future observations of the $v=1 \rightarrow 0 \mathrm{H}_{2}$ emission at sufficiently high spectral resolution $\left(\Delta \lambda / \lambda=10^{-4}\right)$ to measure line profiles. We expect this emission to occur over a velocity range of $\sim 100 \mathrm{~km} \mathrm{~s}^{-1}$. Toward the geometric center of the shell the $\mathrm{H}_{2}$ intensity profile will exhibit two peaks at LSR velocities of $9 \pm 60 \mathrm{~km} \mathrm{~s}^{-1}$, while toward the edges of the shell it will peak near $9 \mathrm{~km} \mathrm{~s}^{-1}$ and has a narrower width. Of course, since the $\mathrm{H}_{2} v=$ 
$1 \rightarrow 0$ emission is so temperature-sensitive and since the gas is likely to be inhomogeneous between the near and far sides of the shell, it is unlikely that the emission will be symmetrical about $v_{\text {LSR }}=9 \mathrm{~km} \mathrm{~s}^{-1}$. Moreover, the emission from the far side will be attenuated by dust within the interior of the envelope. Gautier et al. (1976) indicate that the centroid of the $\mathrm{H}_{2}$ emission at two positions occurs at an LSR velocity of $\sim-7 \mathrm{~km} \mathrm{~s}^{-1}$. The displacement of this centroid from $9 \mathrm{~km} \mathrm{~s}^{-1}$ (the centroid of the broad-band $\mathrm{CO}$ emission) could result from such an asymmetry between the near and far sides of the shell.

From the numerical model we estimate the kinetic energy of the flow to be $4 \times 10^{47} \mathrm{ergs}$, about $10^{-3}$ the kinetic energy of a supernova. The total energy of the event is probably many times greater, as much of the kinetic energy could have been liberated into heat. This event, however, is not likely to cause a catastrophic disruption of the Orion Molecular Cloud. If we take the mass of the cloud to be $\geq 10^{4} M_{\odot}$, then the total momentum of the flow $\left(\sim 2.5 \times 10^{41} \mathrm{~g} \mathrm{~cm} \mathrm{~s}^{-1}\right.$ from the model), when shared over the entire cloud, generates an average velocity of $\leq 1 / 8 \mathrm{~km} \mathrm{~s}^{-1}$.

We thank Dr. Philip Solomon for first mentioning the broadband emission to us. We also thank Adair Lane for much help in obtaining these data. John Kwan thanks the State University of New York for a summer fellowship. Nick Scoville is partially supported by NSF grant MPS73-04949 and a Faculty Research Fellowship at the University of Massachusetts. This is contribution no. 215 of the Five College Observatories.

\section{REFERENCES}

Dickinson, D. F., Gottlieb, C. A., Gottlieb, E. W., and Litvak, M. M. 1976, $A$ p. J., 206, 79.

Gautier, T. N., Fink, U., Treffers, R. R., and Larson, H. P. 1976, preprint.

Gottlieb, C. A., and Ball, J. 1973, Ap. J. (Letters), 184, L59.

Joyce, R., and Grasdalen, G. 1976, private communication.

Kuiper, T. B. H., Knapp, G. R., Knapp, S. L., and Brown, R. L. 1976, A p. J., 408, 19.

Kuiper, T. B. H., Zuckerman, B., and Kuiper, E. N. R. 1975, Bull. $A A S, 7,466$.

Snyder, L. E., Hollis, J. M., Ulich, B. L., Lovas, F. J., Johnson, D. R., and Buhl, D. 1975, A p. J. (Letters), 198, L81.
Sullivan, W. T. 1971, Ph.D. thesis, University of Maryland, College Park.

Thaddeus, P., Kutner, M. L., Penzias, A. A., Wilson, R. W., and Jefferts, K. B. 1972, Ap. J. (Letters), 176, L73.

Ulich, B. L., and Haas, R. W. 1976, Ap. J. Suppl., 30, 247.

Wannier, P. G., Encrenaz, P. J., Wilson, R. W., and Penzias, A. A. $1974, A$ p. J. (Letters), 190, L77.

Wannier, P. G., and Phillips, T. G. 1976, in preparation.

Werner, M. W., Gatley, I., Harper, D. A., Becklin, E. E., Loewenstein, R. F., Telesco, C. M., and Thronson, H. A. 1976, A p. J., 204, 420 .

Note added in proof.-We were informed by a referee that Kuiper, Zuckerman, and Kuiper had also observed the large velocity extent of the CO emission at the Kleinmann-Low nebula (1975). They, however, conclude in favor of a collapsed model.

JoHN Kwan: Bell Laboratories, Murray Hill, NJ 07974

Nick Scoville: Department of Physics and Astronomy, University of Massachusetts, Amherst, MA 01002 